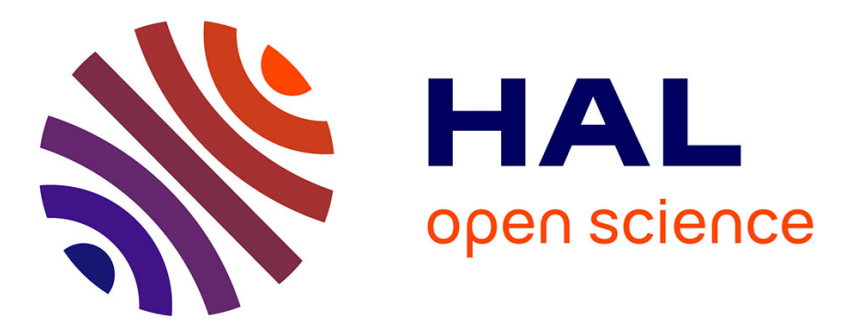

\title{
Refinement indicators for estimating hydrogeologic parameters
}

Mohamed Hedi Riahi, Hend Ben Ameur, Jérôme Jaffré, Rachida Bouhlila

\section{To cite this version:}

Mohamed Hedi Riahi, Hend Ben Ameur, Jérôme Jaffré, Rachida Bouhlila. Refinement indicators for estimating hydrogeologic parameters. [Research Report] RR-8877, INRIA Paris. 2016, pp.25. hal-01285127

\section{HAL Id: hal-01285127 https://inria.hal.science/hal-01285127}

Submitted on 8 Mar 2016

HAL is a multi-disciplinary open access archive for the deposit and dissemination of scientific research documents, whether they are published or not. The documents may come from teaching and research institutions in France or abroad, or from public or private research centers.
L'archive ouverte pluridisciplinaire $\mathbf{H A L}$, est destinée au dépôt et à la diffusion de documents scientifiques de niveau recherche, publiés ou non, émanant des établissements d'enseignement et de recherche français ou étrangers, des laboratoires publics ou privés. 


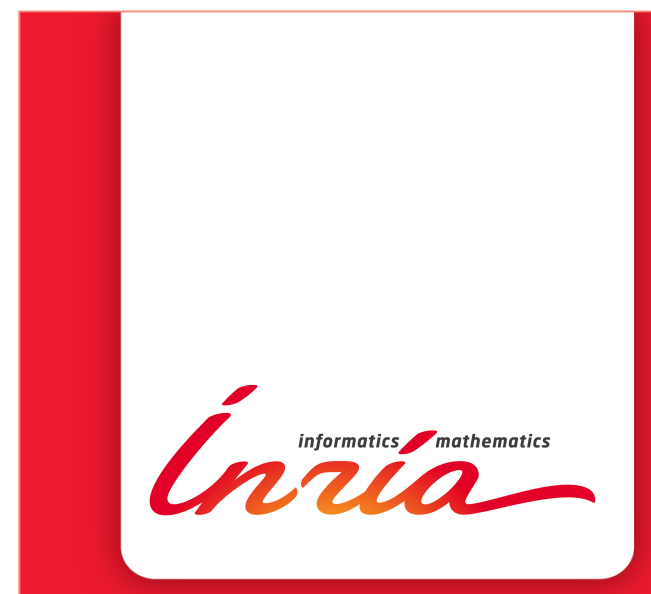

\section{Refinement indicators for estimating hydrogeologic parameters}

Mohamed Hedi Riahi, Hend Ben Ameur , Jérôme Jaffré, Rachida Bouhlila

RESEARCH

REPORT

$\mathrm{N}^{\circ} \mathbf{8 8 7 7}$

February 2016

Project-Team SERENA 



\title{
inzián
}

\section{Refinement indicators for estimating hydrogeologic parameters}

\author{
Mohamed Hedi Riahi*, Hend Ben Ameur †, Jérôme Jaffré $\left.\right|^{\ddagger}$ \\ Rachida Bouhlila $\$$ \\ Project-Team SERENA \\ Research Report n 8877 - February 2016 - 24 pages
}

\begin{abstract}
We identify simultaneously the hydraulic transmissivity and the storage coefficient in a ground water flow governed by a linear parabolic equation. Both coefficients are assumed to be functions which are piecewise constant in space and constant in time. Therefore the unknowns are the coefficient values as well as the geometry of the zones where these parameters are constant. The identification problem is formulated as the minimization of a misfit least-square function. Using refinement indicators, we refine the parameterization locally and iteratively. We distinguish the cases where the two parameters have the same parameterization and different parameterizations.
\end{abstract}

Key-words: Inverse problem, parameter estimation, parameterization, refinement indicators, storage coefficient, hydraulic transmissivity, zonation.

This work was partially supported by the INRIA project Euromed 3+3 Hydrinv.

* Université de Tunis El Manar, Ecole Nationale d'Ingénieurs de Tunis, LAMSIN, BP 37, 1002 Tunis le Belvédère, Tunisia, riahi28@yahoo.fr

† Université de Tunis El Manar, Ecole Nationale d'Ingénieurs de Tunis, LAMSIN, BP 37, 1002 Tunis le Belvédère, Tunisia, hbenameur@yahoo.ca

¥ INRIA Paris, EPI SERENA, BP 105, 78153 Le Chesnay Cedex, France, Jerome. Jaffre@inria.fr

$\S$ Université de Tunis El Manar, Ecole Nationale d'Ingénieurs de Tunis, LMHE, BP 37,1002, Tunis le Belvédère, Tunisia, rjbouhlila@yahoo.fr

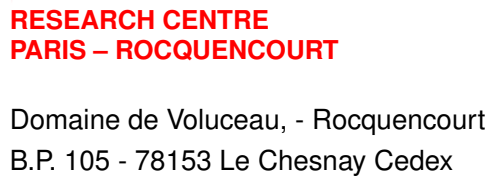




\section{Indicateurs de raffinement pour une paramétrisation optimale dans l'estimation de coefficients hydrogéologiques}

Résumé : On identifie simultanément les coefficients d'emmagasinement et de transmissivité hydraulique dans un écoulement souterrain gouverné par une équation parabolique linéaire. Ces deux paramètres sont supposés être des fonctions constantes par morceaux en espace. Les inconnues du problème sont non seulement les valeurs de ces coefficients mais aussi la geométrie des zones dans lesquelles ces coefficients sont constants. Le problème est formulé comme la minimisation d'une fonction de moindres carrés calculant la différence entre les mesures et les quantités correspondantes évaluées avec la valeur courante des paramètres. L'objectif principal de cet article est la construction d'une technique de paramétrisation adaptative guidée par des indicateurs de raffinement.

Mots-clés : Problème inverse, estimation de paramètres, coefficient d'emmagasinement, transmissivité hydraulique, paramétrisation, indicateurs de raffinement, zonation 


\section{Introduction}

For many hydrogeological problems, such as management and protection of water resources, we need to study the processes of subsurface flow and of transfer of solutes in the subsurface. The corresponding mathematical models involve the hydrological coefficients which characterize different geological zones in the aquifer. Identifying these coefficients by experimental measurements is difficult and have a high cost. In this work, we are interested on solving the inverse problem of identifying these coefficients. There may be one or several unknown coefficients.

The resolution of such inverse problems has been the subject of many studies [16, 14, 8]. The assumption that parameters are constant in each zone is already mentioned in [7] but the set of zones (zonation) was determined by a priori information. The method proposed in this paper consists to build the parameterization in a progressive and iterative way. The first iterative parameterization method is the multiscale method 6, 13. The drawback of this method, where the parameterization is uniformly refined during the iterations, is that it may lead to an overparameterization. The adaptive parameterization method proposed in [1] avoids this drawback since the refinement is local and depends on some "refinement indicator". This method was later used for a scalar parameter identification in [1, 9, 10, 11]. In [2], the parameterization method was extended to the identification of a vector parameter, the colour (Red Green Blue), in a problem of image segmentation .

In this work, we apply the adaptive parameterization method guided by refinement indicators to identify simultaneously the storage coefficient and the hydraulic transmissivity in a confined aquifer which are both unknown. The two coefficients are assumed to be functions piecewise constant in space and constant in time. Therefore they present discontinuities at the interfaces between different geological zones where they are supposed to be constant. The unknowns of our inverse problem are the zonation and the values of the parameters in the zones. We develop different strategies to deal with the cases where the two parameters have the same zonation or different zonations. The developed strategies can be used to identify more then two parameters.

We formulate our inverse problem as a minimization problem of a least-squares function defined as a misfit between measurements and the corresponding quantities computed with "current" parameters. We like to keep as small as possible the number of unknowns in the minimization problem in order to ease the minimization procedure. The data are measurements of the piezometric head in a confined aquifer which is the solution of a linear parabolic equation governing the flow.

The idea of the adaptive parameterization method is to refine a current parameterization according to refinement indicators. A refinement indicator indicates the effect on the optimal data misfit of adding some degree of freedom. The unknown parameterization is estimated, through an iterative process, by refining the scale at which the parameter distribution is described and the process is stopped when the refinement of the scale does not induce a significant decrease of the misfit function. An advantage of this method is to avoid overparameterization. At a given refinement level the parameter is estimated by minimizing the least-squares misfit function. Then we compute refinement indicators and we use these indicators to choose the next refinement level.

This paper is organized as follows. After the introduction in section 1, in section 2 we introduce the direct problem and we set the inverse problems as a minimization problem of a misfit function. Then we present the adjoint method for computing the gradient of that function. In section 3 we present the algorithm of adaptive parameterization and we develop different strategies to identify parameterizations. Finally, in the fourth section we present several numerical experiments corresponding to different versions of the parameterization algorithm including cases where the two parameters have the same zonation or where they have two different 
zonations. The paper ends with concluding remarks.

\section{Problem setting}

We consider the following equations modeling groundwater flow in an isotropic and confined aquifer:

$$
\begin{aligned}
& S \frac{\partial \Phi}{\partial t}-\operatorname{div}(T \nabla \Phi)=Q \quad \text { in } \Omega \times\left(0, t_{f}\right) \\
& \Phi=0 \quad \text { on } \Gamma_{D} \times\left(0, t_{f}\right) \\
& (-T \nabla \Phi) \cdot n \quad=0 \quad \text { on } \Gamma_{N} \times\left(0, t_{f}\right)
\end{aligned}
$$

where $\Omega$ is a bounded connected domain of $\mathbb{R}^{2}$, the time variable $t$ belongs to the interval $\left(0, t_{f}\right)$, $S$ is the storage coefficient and $T$ is the hydraulic transmissivity, $\Phi$ is the piezometric head and $Q$ is a distributed source terms. $n$ is the outer normal to $\Omega, \Gamma_{D}$ and $\Gamma_{N}$ are a partition of the boundary of $\Omega$ denoting respectively Dirichlet and Neumann conditions.

Equations (1) correspond to the direct problem where the unknown is $\Phi$, all other variables, in particular, $S$ and $T$, are known. We are interested on the inverse problem, where $S$ and $T$, are unknown and we have measurements of $\Phi$. This inverse problem is formulated as a minimization problem of a least-square function $J(S, T)$, defined as a misfit between measurements of piezometric head and the piezometric head computed with current parameters $S$ and $T$ :

$$
J(S, T)=\frac{1}{2} \sum_{i=1}^{N_{t}} \sum_{j=1}^{m}\left(\Phi(S, T)\left(t_{i}, M_{j}\right)-d_{i j}^{o b s}\right)^{2}
$$

where $d_{i j}^{o b s}$ is the piezometric head measured at time $t_{i}$ and at point $M_{j}\left(x_{j}, y_{j}\right)$ and $\Phi(S, T)\left(t_{i}, M_{j}\right)$ is the model output for the current coefficients $S$ and $T$ at the same time and the same point.

Estimating the hydraulic transmissivity $T$ and the storage coefficient $S$ amounts to solving

$$
\operatorname{Find}\left(S^{*}, T^{*}\right)=\underset{(S, T) \in U_{a d}}{\arg } \min \quad J(S, T)
$$

where $U_{a d}=\left\{(S, T): S_{\min } \leq S \leq S_{\max } \quad, \quad T_{\min } \leq T \leq T_{\max }\right\}$ is the set of admissible parameters.

Gradient methods are efficient methods for minimizing $J$. A crucial step in the minimization process is the computation of the gradient of the function $J$ with respect to $S$ and $T$. We use the adjoint method [4, 5, 14] to compute this gradient and we present it here briefly. The weak formulation of the direct problem 1 is

$$
\begin{aligned}
& \text { Find } \Phi \in L^{2}\left(0, t_{f} ; H_{0}^{1}(\Omega)\right) \cap C^{0}\left(0, t_{f} ; L^{2}(\Omega)\right) \text { as } \\
& \int_{\Omega} S \partial_{t} \Phi v+\int_{\Omega} T \nabla \Phi \nabla v=\int_{\Omega} Q v \quad \forall v \in H_{0}^{1}(\Omega) \\
& \Phi_{\mid t=0}=\Phi_{0}
\end{aligned}
$$

where $L^{2}\left(0, t_{f} ; H_{0}^{1}(\Omega)\right)$ is the space of square-integrable functions on $\left(0, t_{f}\right)$ with values in $H_{0}^{1}(\Omega)$ and $C^{0}\left(0, t_{f} ; L^{2}(\Omega)\right)$ is the space of continuous functions on $\left(0, t_{f}\right)$ with values in $L^{2}(\Omega)$. 
To minimize $J$ under the constraint (1) we introduce the Lagrangien $\mathcal{L}$ :

$$
\begin{array}{r}
\mathcal{L}\left(S, T ; \Phi, \Phi^{*}\right)=\mathcal{J}(S, T)+\int_{0}^{t_{f}} \int_{\Omega} S \partial_{t} \Phi \Phi^{*} d t+\int_{0}^{t_{f}} \int_{\Omega} T \nabla \Phi \nabla \Phi^{*} d t \\
-\int_{0}^{t_{f}} \int_{\Omega} Q \Phi^{*} d t .
\end{array}
$$

Note that if $\Phi(S, T)$ satisfies (4) then Equation (5) becomes

$$
\mathcal{L}\left(S, T ; \Phi, \Phi^{*}\right)=J(S, T) \quad \forall \Phi^{*} \in H_{0}^{1}(\Omega) .
$$

From Equation $(6)$ it follows

$$
\frac{\partial J}{\partial T} \delta T+\frac{\partial J}{\partial S} \delta S=\frac{\partial \mathcal{L}}{\partial \Phi} \delta \Phi+\frac{\partial \mathcal{L}}{\partial \Phi^{*}} \delta \Phi^{*}+\frac{\partial \mathcal{L}}{\partial T} \delta T+\frac{\partial \mathcal{L}}{\partial S} \delta S .
$$

We choose $\Phi^{*} \in H_{0}^{1}(\Omega)$ such that

$$
\frac{\partial \mathcal{L}}{\partial \Phi}\left(\Phi(S, T), \Phi^{*} ; S, T\right) \delta \Phi=0 \quad \forall \delta \Phi \in H_{0}^{1}(\Omega) .
$$

This choice leads to a simplification of Equation (7). $\Phi^{*}$ is the adjoint state and Equation (8) is the adjoint equation which we can rewrite as

$$
\begin{aligned}
& -S \frac{\partial \Phi^{*}}{\partial t}-\operatorname{div}\left(T \nabla \Phi^{*}\right)=\sum_{i=1}^{N_{t}} \sum_{j=1}^{m}\left(\Phi\left(t_{i}, x_{j}\right)-d_{i j}^{o b s}\right) \quad \operatorname{dans} \Omega \times\left(0, t_{f}\right) \\
& \Phi^{*} \quad=0 \quad \text { in } \Gamma_{D} \times\left(0, t_{f}\right) \\
& \left(-T \nabla \Phi^{*}\right) \cdot n \quad=0 \quad \text { on } \Gamma_{N} \times\left(0, t_{f}\right)
\end{aligned}
$$

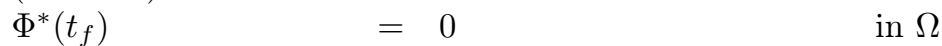

Taking into account Equations (7), (8) the gradient of $J$ can be written as

$$
\begin{aligned}
\frac{\partial J}{\partial T} \delta T+\frac{\partial J}{\partial S} \delta S & =\frac{\partial \mathcal{L}\left(S, T ; \Phi, \Phi^{*}\right)}{\partial T} \delta T+\frac{\partial \mathcal{L}\left(S, T ; \Phi, \Phi^{*}\right)}{\partial S}(H, p ; S, T) \delta S \\
& =\int_{0}^{t_{f}} \int_{\Omega} \delta T \nabla \Phi \nabla \Phi^{*} d \Omega d t+\int_{0}^{t_{f}} \int_{\Omega} \delta S \frac{\partial \Phi}{\partial t} \Phi^{*} d \Omega d t
\end{aligned}
$$

It follows that

$$
\begin{aligned}
& \frac{\partial J}{\partial T}=\int_{0}^{t_{f}} \int_{\Omega} \nabla \Phi \nabla \Phi^{*} d \Omega d t \\
& \frac{\partial J}{\partial S}=\int_{0}^{t_{f}} \int_{\Omega} \frac{\partial \Phi}{\partial t} \Phi^{*} d \Omega d t
\end{aligned}
$$

Equations $(\sqrt{1})$ ) and $(\sqrt{9})$ ) are discretized using the finite element method in space. For the numerical solution of the direct problem 1 and the adjoint problem 9, we use the software SUTRA 15] for simulations of groundwater models in two or three dimensions. The minimization of the misfit function $J$ is obtained by the code N2QN1 [12]. This optimizer implements a quasi-Newton (BFGS) method with line search.

sectionParameterization

Choosing the parameterization is a crucial point in the solution of inverse problems. In hydrogeology many techniques werere developped. As examples close to our problem we cite [7] 
which uses piecewise constant coefficients with a zonation obtained from a priori information, [17] presenting an interpolation method and [3, 13, proposing a multiscale parameterization in which the refinement process is iterative and uniform.

In this work for each coefficient and at each iteration we build a zonation which is a partition of the domain into zones where the parameter is constant and we compute the value of the parameter in each zone. The proposed method will produce an optimal parametrization, in the sense that it minimizes the number of zones, and so the number of unknowns to explain the available data. The zonations can be viewed as discretization grids for the coefficients. If we had used the computational mesh for the coefficient discretization, the inverse problem would have a very large number of unknowns resulting in an optimization problem of very large dimension. There would not be any balance between the amount of data and the number of unknowns and we would not be able to obtain meaningful results.

The idea of our adaptive parameterization method is to refine the zonation iteratively by adding at each iteration only one degree of freedom obtained by dividing one zone of the current zonation into two zones The choice of the zone to be refined and the choice of the refining cut are given by the refinement indicators. The new zone is added where the refinement indicators indicate that it should induce a significant decrease of the misfit function. So the refinement of the parametrization is not arbitrary and not uniform. In this work we suppose that interfaces between zones are carried by the edges of the computational mesh. The refining cuts are thus carried by the edges of mesh cells and belong to a predefined set of curves.
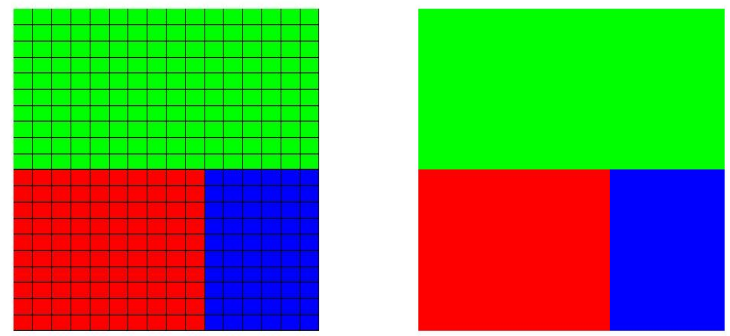

Figure 1: Parametrization and computation mesh (on the left) parametrization and zonation (on the right).

\section{$2.1 \quad$ Refinement indicators}

In this section, following [1, we recall what is a refinement indicator and how it allows to select the refinement which is likely to induce the largest decrease of the misfit function. Let us consider an example where the initial zonation includes only one rectangular zone $Z_{0}$ (see figure 2 ) and we will proceed to its refinement by cutting it into two rectangular zones $Z_{1,1}$ and $Z_{1,2}$.

Let us suppose that the vectorial parameter is constant in the hole domain $\Omega=Z_{0}$, we denote by $m_{0}$ this parameter. In our case $m_{0}=\left(\begin{array}{c}S_{0} \\ T_{0}\end{array}\right)$, where $T_{0}$ is the hydraulic transmissivity and $S_{0}$ is the the storage coefficient. The optimization problem (3) corresponding to this one-zone parameterization $c Z_{0}$ is:

$$
m_{0}^{o p t}=\arg \min _{m_{0} \in U_{a d}} J_{\mathcal{Z}_{0}}\left(m_{0}\right) .
$$



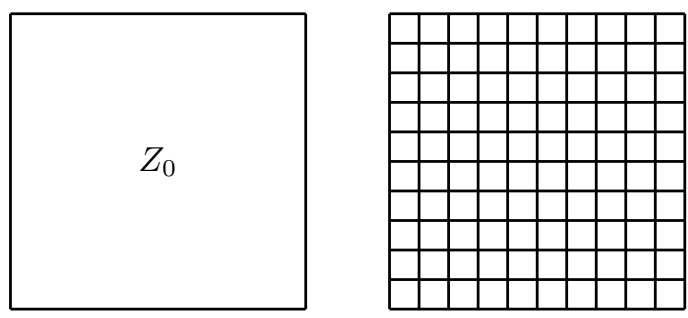

Figure 2: Initial zonation (on the left) and computing mesh (on the right)

Now we want to refine the zonation into a two-zones one with the aim to decrease the misfit function. Each component $S$ and $T$ of the vectorial parameter $m$ may have its own zonation: $\mathcal{Z}_{S 1}, \mathcal{Z}_{T 1}$. We devide $Z_{0}$ into two rectangular zones $Z_{S 1,1}$ et $Z_{S 1,2}$ for the component $S$ and two others $Z_{T 1,1}$ et $Z_{T 1,2}$ for the component $T, Z_{0}=Z_{S 1,1} \cup Z_{S 1,2}=Z_{T 1,1} \cup Z_{T 1,2}$. New zones are separated by the cuts $C_{S 1}$ and $C_{T 1}$. The new zonations are $\mathcal{Z}_{S 1}=\left\{Z_{S 1, i}\right\}_{i=1,2}$ and $\mathcal{Z}_{T 1}=\left\{Z_{T 1, i}\right\}_{i=1,2}$. We denote by $\mathcal{Z}_{1}=\left\{\mathcal{Z}_{S 1}, \mathcal{Z}_{T 1}\right\}$. The unknown of the optimization problem (3) corresponding to the refined zonation is $m_{1}=\left(S_{1,1}, S_{1,2}, T_{1,1}, T_{1,2}\right)^{t}$.
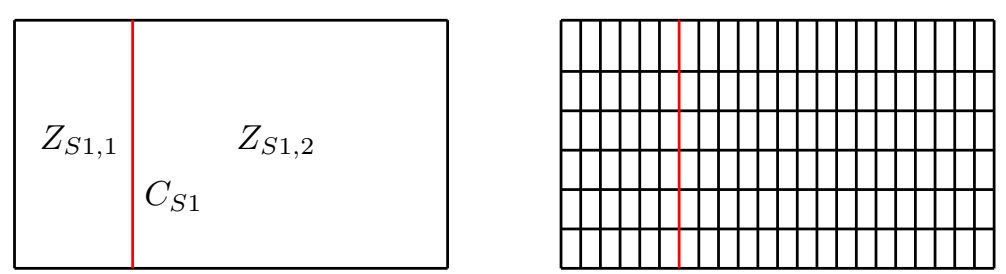

Figure 3: Left: a two-zone zonation $\mathcal{Z}_{S 1}$ for the coefficient $S$. Right: computational mesh for solving the direct and adjoint problems. The cut $C_{S 1}$ is marked in red.

We denote by $c=\left(\begin{array}{c}c_{S} \\ c_{T}\end{array}\right)$ the discontinuities of $S$ and $T$ respectively across $C_{S 1}$ and $C_{T 1}$ :

$$
c_{S}=S_{1,1}-S_{1,2}, \quad c_{T}=T_{1,1}-T_{1,2} .
$$

Introducing the matrix $A=\left(\begin{array}{cccc}1 & -1 & 0 & 0 \\ 0 & 0 & 1 & -1\end{array}\right)$ and assuming that $c$ is known the estimation of $m 1$ comes to solve an optimization problem under the constraint $A m_{1}=c$

$$
m_{1}^{o p t}=\arg \min _{A m_{1}=c} J_{\mathcal{Z}_{1}}\left(m_{1}\right)
$$

where $J_{\mathcal{Z}_{1}}$ is the misfit function corresponding to the new zonation $\mathcal{Z}_{1}$. We note that the minimisation problem 12 is equivalent to 13 when $c=0$.

To the minimization under constraint problem $\sqrt{13}$ we associate the Lagrangian function

$$
L_{\mathcal{Z}_{1}}\left(m_{1}, \lambda\right)=J_{\mathcal{Z}_{1}}\left(m_{1}\right)+\left\langle\lambda_{\mathcal{Z}_{1}}, A m_{1}-c\right\rangle
$$

where $\lambda_{\mathcal{Z}_{1}}=\left(\begin{array}{c}\lambda_{S}, \mathcal{Z}_{1} \\ \lambda_{T, \mathcal{Z}_{1}}\end{array}\right)$ is the Lagrange mutiplier associeted to the contraint $A m_{1}=c$. The 
optimality conditions associated to 13 are then :

$$
\begin{aligned}
& \frac{\partial L_{\mathcal{Z}_{1}}\left(m_{1}^{o p t}, \lambda_{\mathcal{Z}_{1}}^{o p t}\right)}{\partial m_{1}}=\nabla J_{\mathcal{Z}_{1}}\left(m_{1}^{o p t}\right)+A^{t} \lambda_{\mathcal{Z}_{1}}^{o p t}=0 \\
& \frac{\partial L_{\mathcal{Z}_{1}}\left(m_{1}^{o p t}, \lambda_{\mathcal{Z}_{1}}^{o p t}\right)}{\partial \lambda}=A m_{1}^{o p t}-\lambda_{\mathcal{Z}_{1}}^{o p t}=0
\end{aligned}
$$

Taking into account the optimality conditions 15 and deriving the Lagrangian function $L_{\mathcal{Z}_{1}}$ with respect to $c$, we obtain

$$
\left.\frac{\partial L_{\mathcal{Z}_{1}}\left(m_{1}^{o p t}, \lambda_{\mathcal{Z}_{1}}^{o p t}\right)}{\partial c}\right|_{c=0}=\left.\frac{\partial L_{\mathcal{Z}_{1}}\left(m_{1}^{o p t}, \lambda_{\mathcal{Z}_{1}}^{o p t}\right)}{\partial m_{1}}\right|_{c=0} \frac{\partial m_{1}}{\partial c}+\left.\frac{\partial L_{\mathcal{Z}_{1}}\left(m_{1}^{o p t}, \lambda_{\mathcal{Z}_{1}}^{o p t}\right)}{\partial \lambda}\right|_{c=0} \frac{\partial \lambda}{\partial c}=0
$$

Using (14) we deduce

$$
\left.\frac{\partial L_{\mathcal{Z}_{1}}\left(m_{1}^{o p t}, \lambda_{\mathcal{Z}_{1}}^{o p t}\right)}{\partial c}\right|_{c=0}=\left.\nabla_{c} J_{\mathcal{Z}_{1}}\left(m_{1}^{o p t}\right)\right|_{c=0}-\lambda_{\mathcal{Z}_{1}}^{o p t}=0
$$

Then we have $\left.\nabla_{c} J_{\mathcal{Z}_{1}}\left(m_{1}^{o p t}\right)\right|_{c=0}=\lambda_{\mathcal{Z}_{1}}^{o p t}$.

The Taylor expansion of $J_{\mathcal{Z}_{1}}$ as a function of the discontinuity $c$ in a neighborhood of $c=0$ is

$$
\left.J_{\mathcal{Z}_{1}}\left(m_{1}^{o p t}\right)\right|_{c}=\left.J_{\mathcal{Z}_{1}}\left(m_{1}^{o p t}\right)\right|_{c=0}+\left\langle c,\left.\nabla_{c} J_{\mathcal{Z}_{1}}\left(m_{1}^{o p t}\right)\right|_{c=0}\right\rangle+o(\|c\|)
$$

Since $\left.J_{\mathcal{Z}_{1}}\left(m_{1}^{o p t}\right)\right|_{c=0}=J_{0}\left(m_{0}^{o p t}\right)$ we conclude that $\left\langle c,\left.\nabla_{c} J_{\mathcal{Z}_{1}}\left(m_{1}^{o p t}\right)\right|_{c=0}\right\rangle=\left\langle c, \lambda_{\mathcal{Z}_{1}}^{\text {opt }}\right\rangle$ modelize, at a first order, the variation between $J_{\mathcal{Z}_{1}}\left(m_{1}^{\text {opt }}\right)$ and $J_{\mathcal{Z}_{0}}\left(m_{0}^{\text {opt }}\right)$ in a neighborhood of $c=0$.

Components of $\lambda_{\mathcal{Z}_{1}}^{\text {opt }}$ give two refinement indicators $I_{S}^{\mathcal{Z}_{1}}, I_{T}^{\mathcal{Z}_{1}}$ corresponding respectively to the cuts $C_{S 1}$ and $C_{T 1}$ inducing the zonation $\mathcal{Z}_{1}$. Without solving the optimization problem 13 computing $\lambda_{\mathcal{Z}_{1}}^{\text {opt }}$ allows to conclude if the zonation refinement induced by the cuts $C_{S 1}$ et $C_{T 1}$ would produce a significant decrease of the misfit function or not.

The Lagrange multiplier $\lambda^{o p t}$ is computed using Equation $\left[15\right.$. Then $\lambda_{\mathcal{Z}_{1}}^{o p t}=\left(\lambda_{S, \mathcal{Z}_{1}}^{o p t}, \lambda_{T, \mathcal{Z}_{1}}^{o p t}\right)$ where

$$
\lambda_{S, \mathcal{Z}_{1}}^{o p t}=\frac{\partial J_{\mathcal{Z}_{1}}}{\partial S_{1,1}}\left(m_{1}^{o p t}\right)=-\frac{\partial J_{\mathcal{Z}_{1}}}{\partial S_{1,2}}\left(m_{1}^{o p t}\right), \quad \lambda_{T, \mathcal{Z}_{1}}^{o p t}=\frac{\partial J_{\mathcal{Z}_{1}}}{\partial T_{1,1}}\left(m_{1}^{o p t}\right)=-\frac{\partial J_{\mathcal{Z}_{1}}}{\partial T_{1,2}}\left(m_{1}^{o p t}\right) .
$$

We note that for the computation of the refinement indicator we use the gradient of $J_{\mathcal{Z}_{1}}$ with respect to the values of the coefficients in the zones. This gradient can be easily calculated from "the fine gradient" computed on the computing mesh:

$$
\frac{\partial J_{\mathcal{Z}_{1}}}{\partial S_{1, i}}\left(m_{1}^{o p t}\right)=\sum_{K \subset Z_{S 1, i}} \frac{\partial J_{\mathcal{Z}_{1}}}{\partial S_{K}}\left(m_{1}^{o p t}\right), \quad \frac{\partial J_{\mathcal{Z}_{1}}}{\partial T_{1, i}}\left(m_{1}^{o p t}\right)=\sum_{K \subset Z_{T 1, i}} \frac{\partial J_{\mathcal{Z}_{1}}}{\partial T_{K}}\left(m_{1}^{o p t}\right), i=1,2
$$

where $K$ denotes a cell of the computing mesh. Thus using the adjoint method (see Section 2 ) is crucial for an efficient computation of the gradient and of the refinement indicators.

In this work, to refine a zone by dividing it into two zones we use only vertical and horizontal cuts carried by the computational mesh edges (see Figure 4). Other cuts are possible (see [1]). 

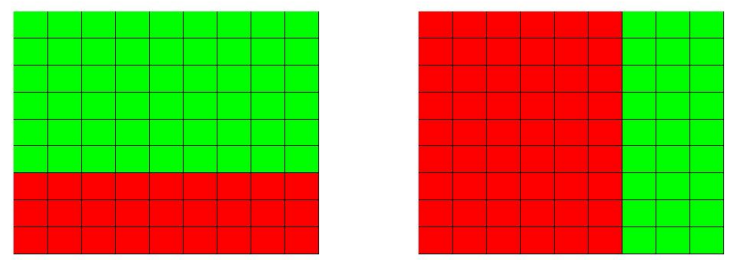

Figure 4: horizontal cut (on the left) and vertical cut (on the right).

\subsection{Algorithm}

We are adapting an algorithm initially proposed in [1] for the estimation of a scalar parameter in porous media and extended in [2] to image segmentation with a vector parameter whose components are the three colors Red,Green,Blue. In image segmentation the direct problem is just the identity model, while for the hydrogeological problem, the model is given by (1) which require the solution of a PDE. Therefore the application of the adaptive parameterization algorithm is much more complicated.

We choose an initial zonation $\mathcal{Z}_{0}=\Omega$. A step of the algorithm is as follows:

1. Given a zonation $\mathcal{Z}_{k-1}=\left(\mathcal{Z}_{S, k-1}, \mathcal{Z}_{T, k-1}\right)$ with $k$ zones for each parameter, minimize $J_{\mathcal{Z}_{k-1}}$ to obtain an optimal parameter $m_{k}^{o p t}$.

2. Build a new zonation $\widetilde{\mathcal{Z}}_{k}=\left(\widetilde{\mathcal{Z}}_{S, k}, \widetilde{\mathcal{Z}}_{T, k}\right)$ with $k+1$ zones for each parameter, by introducing a cut dividing a zone into two zones following four steps:

(a) Consider $\mathcal{C}^{a d}$ the set of cuts $C$ giving a possible zonation $\widetilde{\mathcal{Z}}_{k}^{C}$ having $k+1$ zones for each parameter obtained by dividing into two zones only one zone in $\mathcal{Z}_{S, k-1}$ and only one zone in $\mathcal{Z}_{T, k-1}$.

(b) For each $C \in \mathcal{C}^{a d}$ we compute $\lambda_{\widetilde{\mathcal{Z}}_{k}^{C}}^{o p t}=\left(\lambda_{S, \widetilde{\mathcal{Z}}_{k}^{C}}^{o p t}, \lambda_{T, \widetilde{\mathcal{Z}}_{k}^{C}}^{o p t}\right)$ using (17), 18).

(c) For each zone $\widetilde{\mathcal{Z}}_{k}^{C}, C \in \mathcal{C}^{a d}$, compute a refinement indicator $\widetilde{I}_{k}^{C}$ from vectors $\lambda_{\widetilde{\mathcal{Z}}_{k}^{C}}^{\text {opt }}$ according to a choosen strategy proposed in the following Section 2.3

(d) Select a set of zonations $\widetilde{\mathcal{Z}}_{k}^{C}, C \in \mathcal{C}_{k} \subset \mathcal{C}^{a d}$ corresponding to the largest values of $\widetilde{I}_{k}^{C}$ and for each of these zonations minimize $J_{\widetilde{\mathcal{Z}}_{k}}$.

3. Update the zonation by taking fo each $\mathcal{Z}_{k}$ the zonation $\widetilde{\mathcal{Z}}_{k}^{C}$ which produces the largest decrease of the misfit function.

The stopping criteria for the algorithm are:

- The value of the misfit function is close to zero.

- The misfit function stops decreasing during a number of iterations.

- The values of the refinement indicators are small.

- The norm of the gradient of the misfit function is small. 


\subsection{Strategies for the computation of the refinement indicators}

In this section, inspired by [2, we develop different strategies for the computation of refinement indicators $\widetilde{I}_{k}^{C}$ from the Lagrange mutiplier $\lambda_{\widetilde{\mathcal{Z}}_{k}^{C}}^{\text {opt }}$ computed in 117 , 18.

\subsubsection{Strategy 1: euclidean norm for refinement indicators}

In this strategy we assume that the two coefficients $S$ and $T$ have the same zonation, we define the refinement indicators by

$$
\widetilde{I}_{k}^{C}=\sqrt{\left(\lambda_{S, \widetilde{\mathcal{Z}}_{k}^{C}}^{o p t}\right)^{2}+\left(\lambda_{T, \widetilde{\mathcal{Z}}_{k}^{C}}^{o p t}\right)^{2}} .
$$

Since we have a scalar refinement indicator we can apply the adaptive parametrization algorithm defined in the scalar case [1. At each iteration we perform the following steps:

1. Compute $I_{k}^{\max }=\max _{C \in \mathcal{C}^{a d}} \widetilde{I}_{k}^{C}$.

2. Introduce the subset of cuts $\mathcal{C}_{\delta}^{a d}=\left\{C \in \mathcal{C}^{a d}: \widetilde{I}_{k}^{C} \geq \delta * I_{k}^{\max }\right\}$ to $0.5 \leq \delta \leq 1$.

3. Minimize the misfit function for each partition corresponding to a cut $C \in \mathcal{C}_{\delta}^{a d}$.

4. Keep only the cut $C^{*}$ which induces the largest decrease of the misfit function.

This strategy is efficient if both parameters $S$ and $T$ have the same zonation.

\subsubsection{Strategy 2: best cut for all parameters}

In this strategy the algorithm is guided by two refinement indicators, one for each coefficient $S$ and $T$. We select two sets of possible cuts according to the two indicators. During an iteration, a temporary selection is made with respect to only one of the two parameters. At the end of the iteration the same cut is applied to both parameters. It is chosen according to the criterion of "best decrease" of the misfit function. At each iteration we start by the following steps:

1. Compute the refinement indicators: $I_{S, k}^{\max }=\max _{C \in \mathcal{C}_{S}^{a d}} \lambda_{S, \widetilde{\mathcal{Z}}_{k}^{c}}^{o p t}, I_{T, k}^{\max }=\max _{C \in \mathcal{C}_{T}^{a d}} \lambda_{T, \widetilde{\mathcal{Z}}_{k}^{\text {opt }}}^{\text {. }}$

2. Introduce the subset of cuts $\mathcal{C}_{S \delta}^{a d}=\left\{C \in \mathcal{C}_{S}^{a d}: \widetilde{I}_{S, k}^{C} \geq \delta * I_{S, k}^{\max }\right\}$ to $0.5 \leq \delta \leq 1$.

3. Introduce the subset of cuts $\mathcal{C}_{T \delta}^{a d}=\left\{C \in \mathcal{C}_{T}^{a d}: \widetilde{I}_{T, k}^{C} \geq \delta * I_{T, k}^{\max }\right\}$ to $0.5 \leq \delta \leq 1$.

4. Minimize the misfit function freezing the zonation for $T$ at $\mathcal{Z}_{T, k-1}$ and using as zonation for $S$, the zonations $\widetilde{\mathcal{Z}}_{S, k}^{C}$ where $C \in \mathcal{C}_{S}^{a d}$. Keep only the cut $C_{S}^{*}$ that maximizes the decrease of the misfit function.

5. Minimize the misfit function by freezing the zonation for $S$ at $\mathcal{Z}_{S, k-1}$ and using as zonation for $T$, the zonations $\widetilde{\mathcal{Z}}_{T, k}^{C}$ where $C \in \mathcal{C}_{T}^{a d}$. Keep only the cut $C_{T}^{*}$ that maximizes the decrease of the misfit function.

Then, after obtaining a cut for each parameter, we apply the following steps:

1. Minimize the misfit function with the zonation obtained by applying the cut $C_{S}^{*}$ for both parameters $S$ and $T$. 
2. Minimize the misfit function with the zonation obtained by applying the cut $C_{T}^{*}$ for both parameters $S$ and $T$.

3. Keep for the two parameters the cut $C^{*}=C_{S}^{*}$ or $C_{T}^{*}$ which induces the largest decrease of the misfit function.

Thus, going from one iteration to the next, we introduce the same cut for $S$ and $T$.

\subsubsection{Strategy 3: best cut for each parameter}

In this strategy, we treat the parameters $S$ and $T$ independently. The choice of this strategy is justified by the case where two geological zones for the porosity and the permeabilities are different. The zonations are constructed in the following way.

1. Compute the refinement indicators: $I_{S, k}^{\max }=\max _{C \in \mathcal{C}_{S}^{a d}} \lambda_{S, \widetilde{\mathcal{Z}}_{k}^{C}}^{o p t}, I_{T, k}^{\max }=\max _{C \in \mathcal{C}_{T}^{a d}} \lambda_{T, \widetilde{\mathcal{Z}}_{k}^{C}}^{o p t}$.

2. Introducing the subset of cuts $\mathcal{C}_{S \delta}^{a d}=\left\{C \in \mathcal{C}_{S}^{a d}: \widetilde{I}_{S, k}^{C} \geq \delta * I_{S, k}^{\max }\right\}$ to $0.5 \leq \delta \leq 1$.

3. Introduce the subset of cuts $\mathcal{C}_{T \delta}^{a d}=\left\{C \in \mathcal{C}_{T}^{a d}: \widetilde{I}_{T, k}^{C} \geq \delta * I_{T, k}^{\max }\right\}$ to $0.5 \leq \delta \leq 1$.

4. Minimize the misfit function by freezing the zonation for $T$ at $\mathcal{Z}_{T, k-1}$ and we use as zonation for $S$, the zonation $\widetilde{\mathcal{Z}}_{S, k}^{C}$ for $C \in \mathcal{C}_{S}^{a d}$. Keep only the cut $C_{S}^{*}$ that maximizes the decrease of the misfit function.

5. Minimize the misfit function by freezing the zonation for $S$ at $\mathcal{Z}_{S, k-1}$ and we use as zonation for $T$, the zonation $\widetilde{\mathcal{Z}}_{T, k}^{C}$ for $C \in \mathcal{C}_{T}^{a d}$. Keep only the cut $C_{T}^{*}$ that maximizes the decrease of the misfit function.

6. The new zonation is $\mathcal{Z}_{k}=\left(\widetilde{\mathcal{Z}}_{S, k}^{C_{S}^{*}}, \widetilde{\mathcal{Z}}_{T, k}^{C_{T}^{*}}\right)$.

\section{$3 \quad$ Numerical experiments}

We consider as an example the aquifer of Rocky Mountain Arsenal, Denver, Colorado in USA [15] represented in Figure 5. This aquifer is horizontal and rectangular. The computational domain is discretized with a mesh of 320 square elements and 357 nodes. The thickness of the aquifer is equal to $40 \mathrm{ft}$. In this aquifer, there are four wells, three pumping wells with a constant rate $Q_{o u t}=-0.2 \mathrm{ft}^{-3} / \mathrm{s}$ and an injection well with a constant rate $Q_{I N}=10 \mathrm{ft}^{-3} / \mathrm{s}$ (see Figure 5. The lateral boundaries are impermeable. On the top boundary, the piezometric head is set constant and equal to $250.0 \mathrm{ft}$. On the bottom boundary, the piezometric head is varying linearly from left to right from $17.5 \mathrm{ft}$ to $57,5 \mathrm{ft}$.

In the first following numerical experiences, data are numerical measurements of the piezometric head at each node of the computing mesh and at each time step. In later experiments the number of measurements will be reduced. These measurements will be observations of the numerical solution obtained with the exact coefficients.

The exact hydraulic transmissivity and storage coefficient are constant in the whole aquifer with values $T=2.5 \cdot 10^{-4} \mathrm{~m} / \mathrm{s}, S=6.0010^{-7}$, except in inclusions where their values are $T=$ 2.5.10 $0^{-6} \mathrm{~m} / \mathrm{s}, S=9.9510^{-7}$. We consider a case with the same zonation for both coefficients, with two inclusions as shown in Figures 5, 6. We will also consider a case where each coefficient has its own inclusion as in Figure 11. We will compare results obtained with the three different strategies for computing the refinement indicators presented in section 2.3 


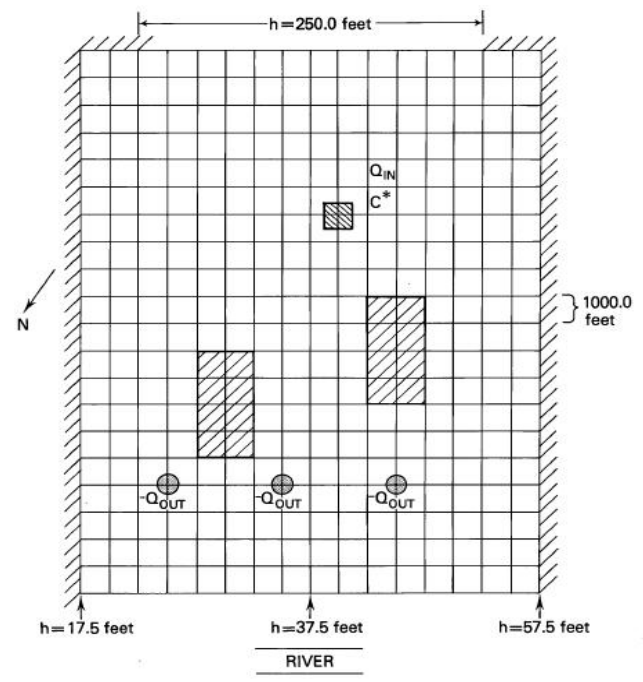

Figure 5: Geometry of the Rocky Mountain aquifer .

\subsection{Experiments using the euclidean norm for refinement indicators (strategy 1)}

In a first test we consider exact coefficients $S$ and $T$ with the same zonation shown In Figure 6 which shows also the computational mesh. Figure 7 shows the piezometric head computed with the exact coefficients which is used as data $d^{\text {obs }}$. We observe two depressions corresponding to the two inclusions with very low permeability compared to the surrounding domain and we notice a peak located at the injection well. We identify the zonation of the coefficients $S$ and $T$ and

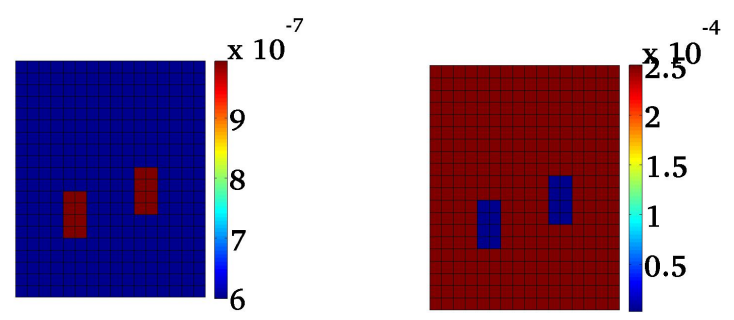

Figure 6: Test 1: exact coefficients $S$ (left) and $T$ (right).

their values in each zone. We use the algorithm described in section 2.2 with strategy 1 where the refinement indicator is defined as the euclidean norm of the vector indicator $I=\left(I_{S}, I_{T}\right)$. We start with a parameterization where the two coefficients $S$ and $T$ are constant in the whole domain (Figure 8). With strategy 1 the zonation is the same for both coefficients at all iterations. Going from one iteration to the next, the current zonation for the two coefficients is refined by introducing one cut corresponding to the largest decrease of the misfit function. Figure 9 shows the evolution of the coefficient values during the iterations of the algorithm. It should be noted that colors correspond to coefficient values and do not represent the zones. If a coefficient has the same value in two different adjacent zones these zones have the same color but a change 


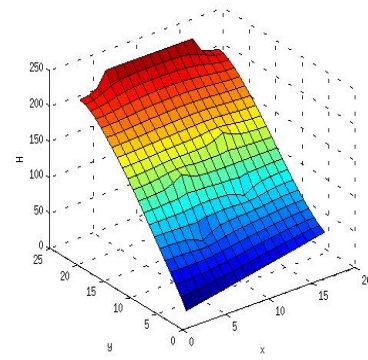

Initial time

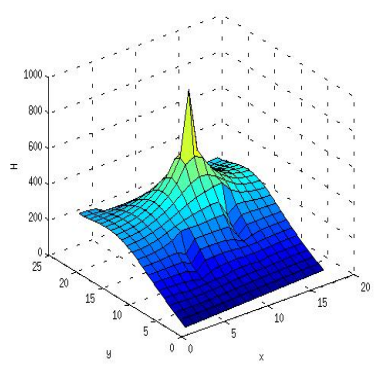

After 6 days

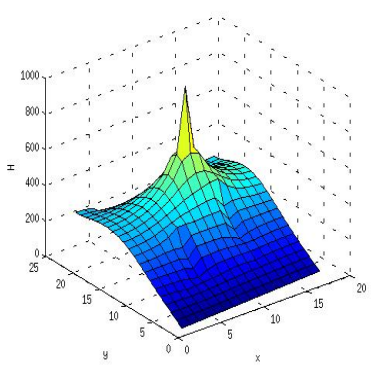

Final time

Figure 7: Test 1: piezometric head at 3 times calculated with the coefficients shown in Figure 6 .
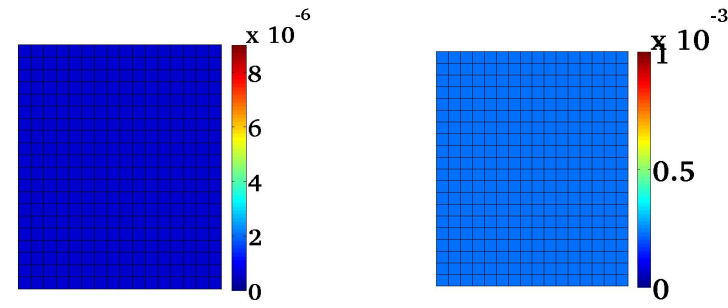

Figure 8: Test 1: optimal values of $S$ (left) and $T$ (right) after the first minimization.

of color corresponds to a change of zone. We remark that in the third iteration, we already identified edges limiting a large zone sourrounding the two inclusions that we are looking for. At the 9th iteration, we observe that both sought inclusions start to appear and they are identified at iteration 14. The exact parameter values are obtained at the 16 th iteration. Note that the exact $T$ is obtained faster than the storage coefficient. This behavior will be observed in all experiments and is due to a smaller sensitivity of the system to $S$ than to $T$. 

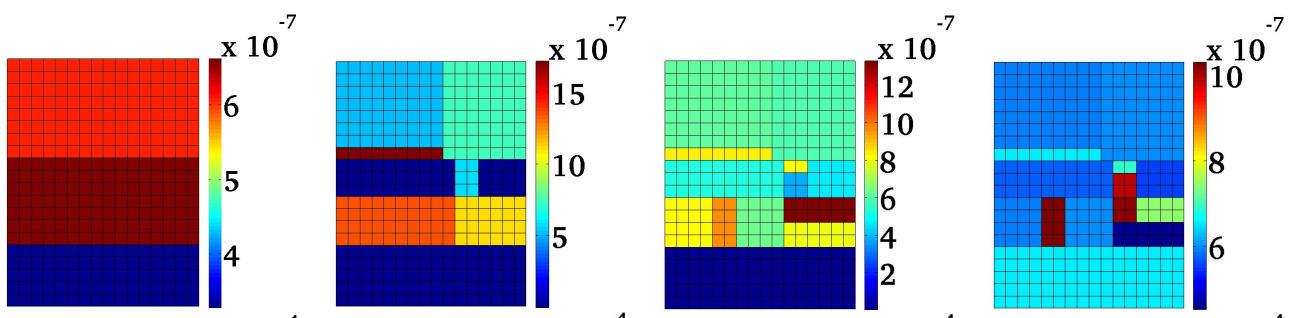

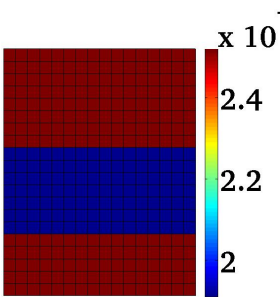

Iteration 3

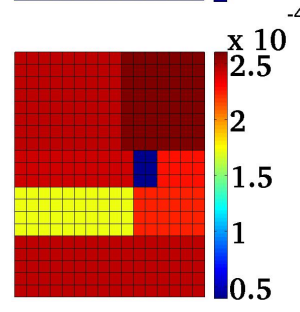

Iteration 9

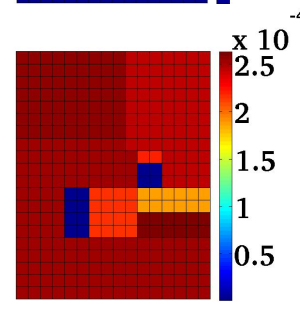

Iteration 13

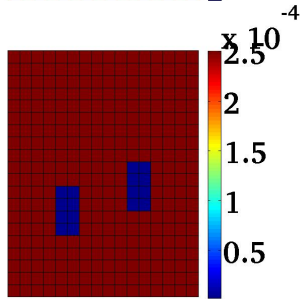

Iteration 14

Figure 9: Test 1: coefficients $S$ (left) and $T$ (right) computed during the iterations with strategy 1 , when exact $S$ and $T$ have the same zonation.

Figure 10 shows the final zonation with 16 zones and the variation of the normalized misfit function during the iterations.
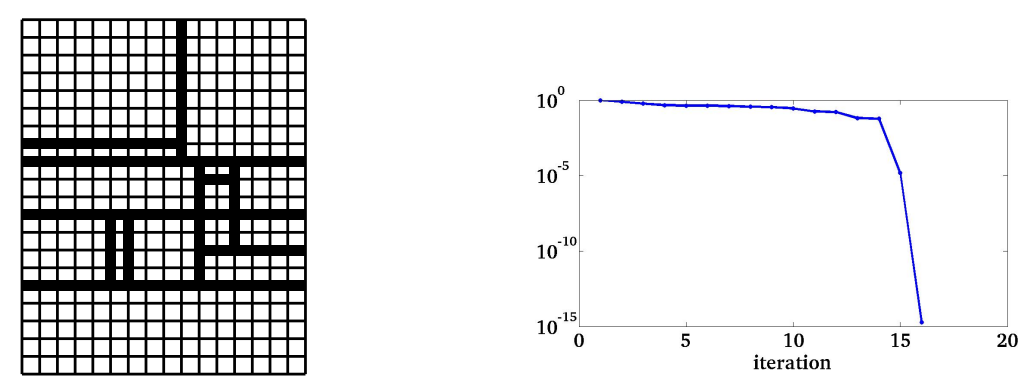

Figure 10: Test 1: strategy 1 when exact $S$ and $T$ have the same zonation. Final zonation for $S$ and $T$ and variation of the misfit function during the iterations with strategy 1.

Now we would like to see what happens if we apply the same strategy in a case where the exact coefficients have different zonations. Therefore we consider coefficients given as in Figure 11. The values of the exact coefficients are the same as in test 1 but the zonation differs since the inclusion is different for each coefficient.

We initialize the algorithm with a parametrization where the two coefficients are constant in the whole domain (Figure 12). Figure 13 shows the values of the coefficients obtained during the iterations. The algorithm identifies $T$ at iteration 17 but then fails to identify $S$. 

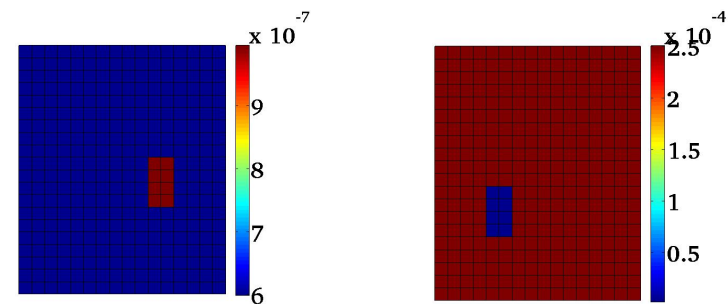

Figure 11: Test 2: exact coefficients $S$ (left) and $T$ (right) with different zonations.

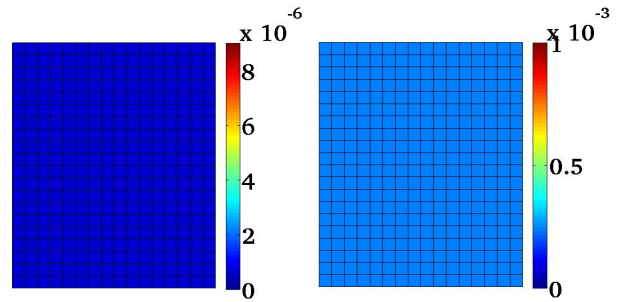

Figure 12: Test 2: optimal values of $S$ (left) and $T$ (right) after the first optimization.
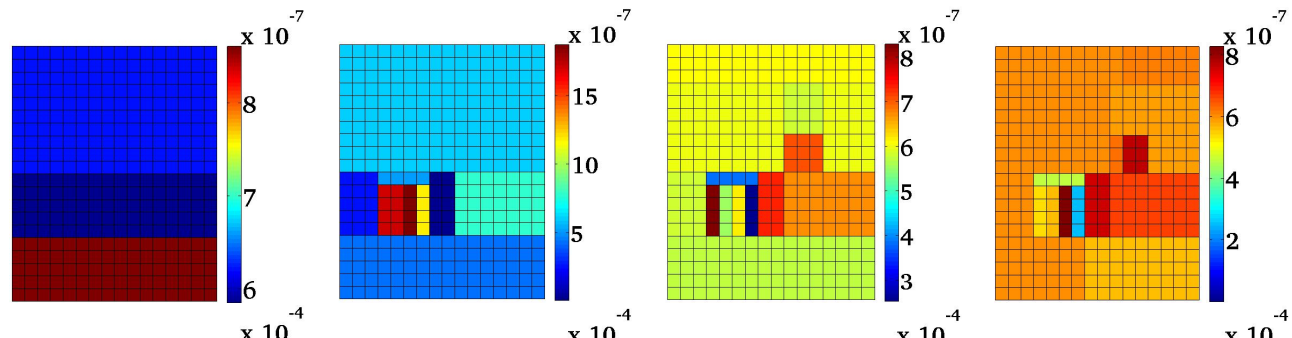

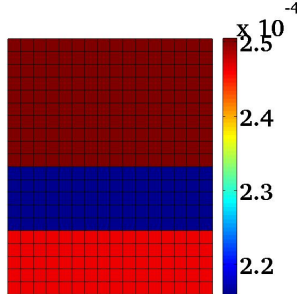

Iteration 3

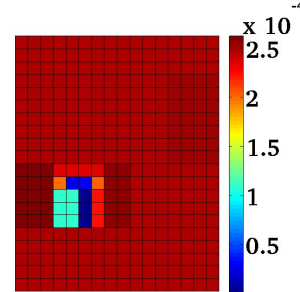

Iteration 14

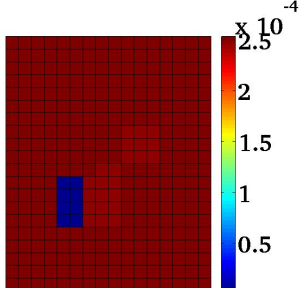

Iteration 17

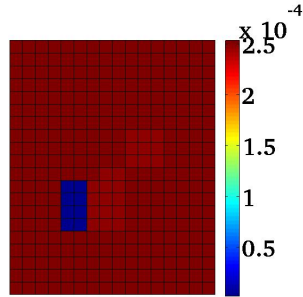

Iteration 21

Figure 13: Test 2: coefficients $S$ (top row) and $T$ (bottom row) computed during the iterations with strategy 1 , when exact $S$ and $T$ have different zonations.

Figure 14 shows the final zonation for the two coefficients after 20 iterations and the variation of the normalized misfit function during the iterations.

$\mathrm{RR} \mathrm{n}^{\circ} 8877$ 

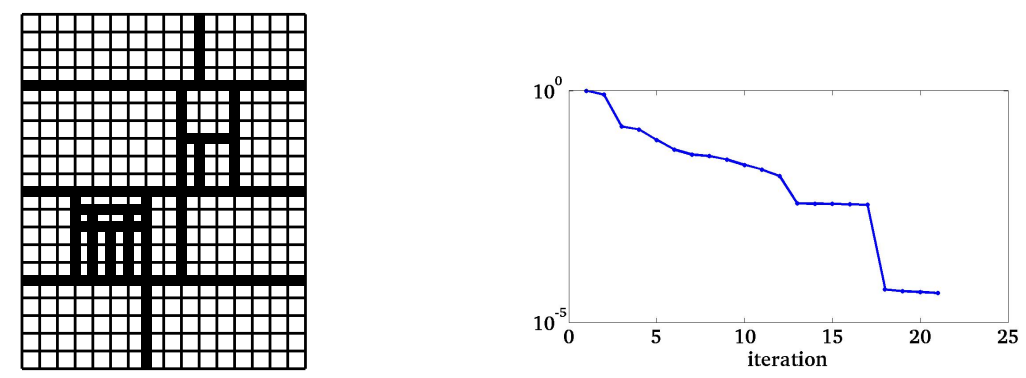

Figure 14: Test 2: strategy 1 when exact $S$ and $T$ have different zonations. Zonation after 20 iterations (left) and variation of the misfit function during the iterations (right).

\subsection{Experiments using the best cut for the two coefficients (strategy 2)}

In this section we take the same data as in tests 1 and 2 but we apply strategy 2: going from one iteration to the next, we add the same cut for both coefficients corresponding to the largest decrease of the misfit function. Again strategy 2 calculates the same zonation for both coefficients.

Test 3 corresponds to the case where the two exact coefficients have the same zonation (Figure 6 and test 4 corresponds to the case where the two exact coefficients have different zonations (Figure 11). Initializations are the same as in test 1 and test 2, with constant coefficients over the whole domain (Figures 8 and 12 .

For test 3 Figure 15 gives the evolution of the computed coefficients during the iterations. It took 24 iterations to obtain the exact coefficients, a few more iterations than in test 1. Again $T$ was identified first. Figure 16 shows the final zonation obtained after 24 iterations and the variation of the normalized misfit function during the iterations.

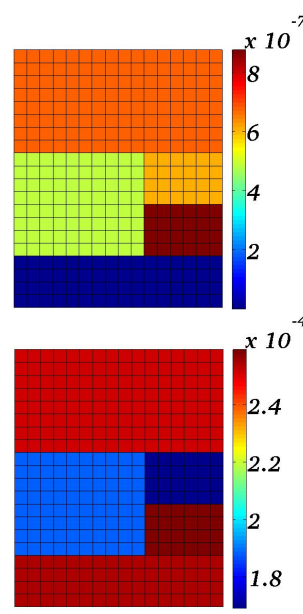

Iteration 5
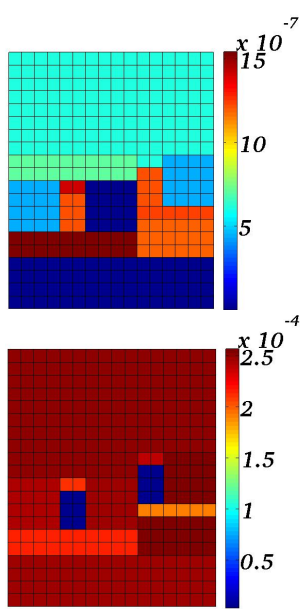

Iteration 13
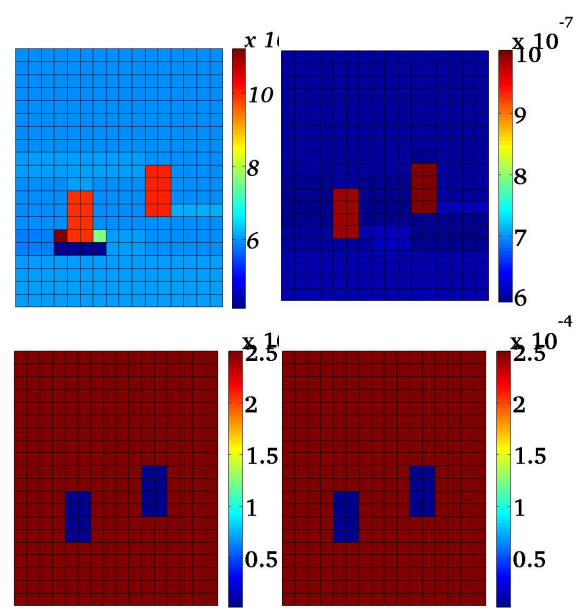

Iteration 20

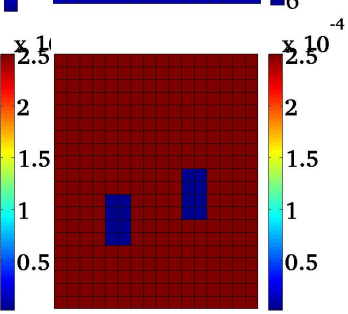

Iteration 24

Figure 15: Test 3: coefficients $S$ (top row) and $T$ (bottom row) computed at iteration 24 using strategy 2, when exact $S$ and $T$ have the same zonation.

We apply now strategy 2 to the case where the two coefficients have different zonations. As before, the algorithm is initialized with a parameterization in which the two coefficients are 

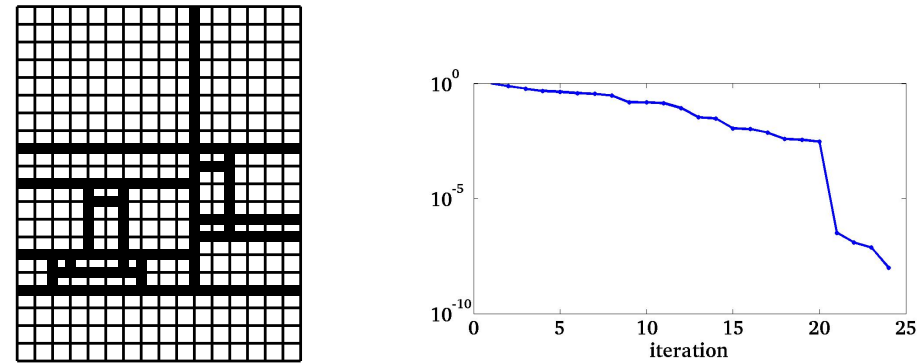

Figure 16: Test 3: zonation after 24 iterations for the two coefficients and variation of the misfit function during the iterations.

constant. The initial values of the two coefficients are shown in figure 12. Figure 17] shows the variation of the computed coefficients during the iterations. At iteration 10 we identify the zonation and the exact values for the hydraulic transmissivity. For the storage coefficient, it took more iterations to find its zonation and its value. Like for other tests before this is due to the smaller sensitivity of the system to this coefficient. Figure 18 shows the zonation for the two

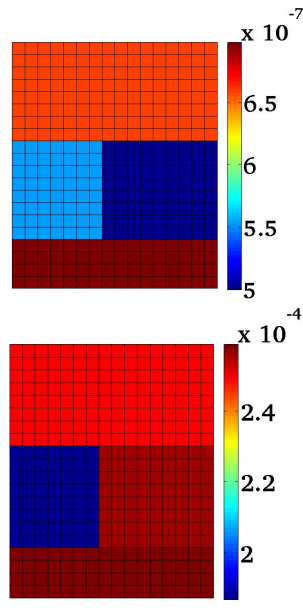

Iteration 4
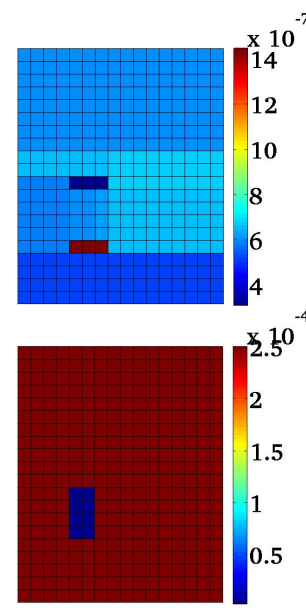

Iteration 10

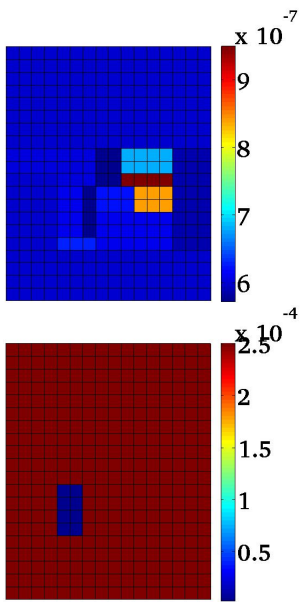

Iteration 18

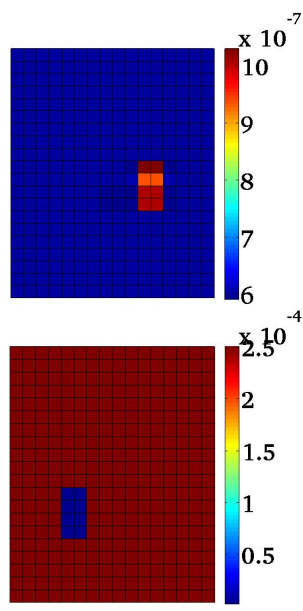

Iteration 29

Figure 17: Test 4: coefficients $S$ (on the left) and $T$ (on the right) computed during the iterations using strategy 2 , when exact $S$ and $T$ have different zonations.

calculated coefficients and the variation of the normalized misfit function during the iterations.

\subsection{Experiments using the best cut for each parameter (strategy 3)}

The main feature of this strategy is that during the algorithm iterations we treat the two coefficients independently. Each coefficient has its own zonation constructed with its own refinement indicator as described in section 2.3.3. We start with the case where the two coefficients have the same zonation 6. Figure 19 shows the variation of the computed coefficients during the iterations. Figure 20 shows zonations for $S$ and $T$ after 28 iterations and the variation of the normalized misfit function during the iterations. The algorithm identifies $T$ after 24 iterations but fails to identify $S$. 

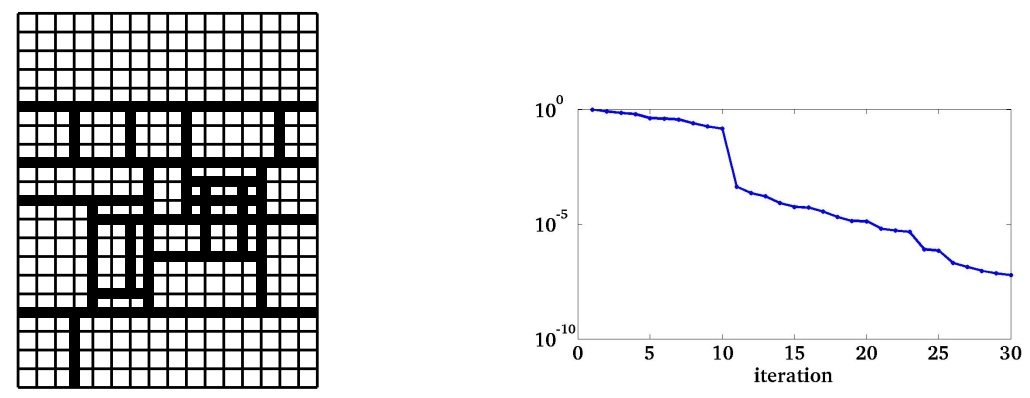

Figure 18: Test 4: strategy 2 when the two exact coefficients have different zonations. Zonation for $S$ and $T$ after 29 iterations (left) and variation of the misfit function during the iterations (right).

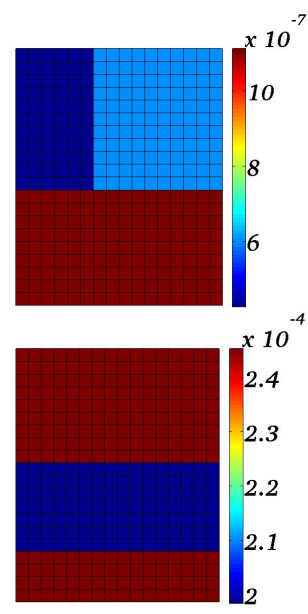

Iteration 3

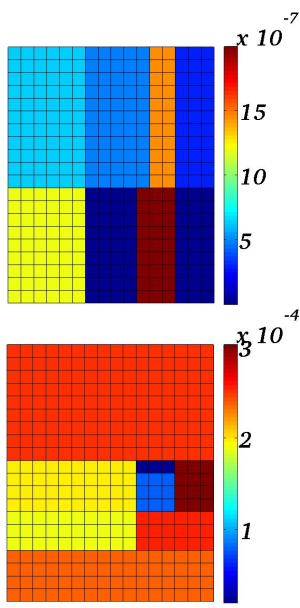

Iteration 8

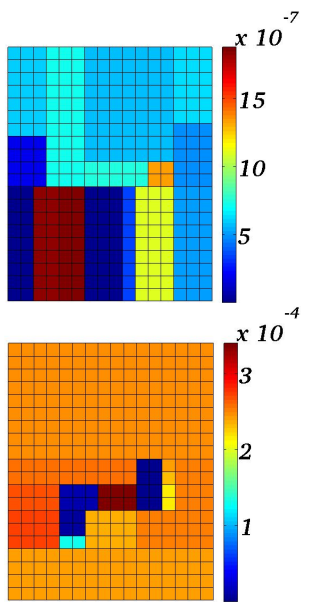

Iteration 19

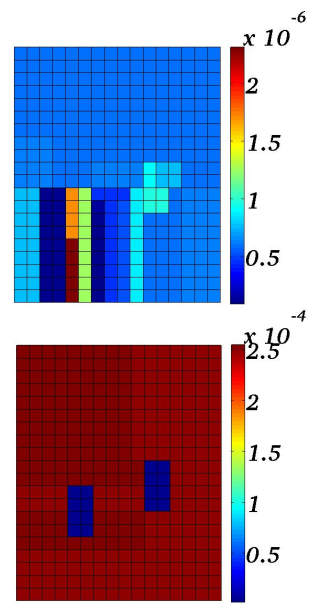

Iteration 25

Figure 19: Test 5: coefficients $S$ (on the left) and $T$ (on the right) computed during the iterations using strategy 3 , when exact $S$ and $T$ have the same zonation. 

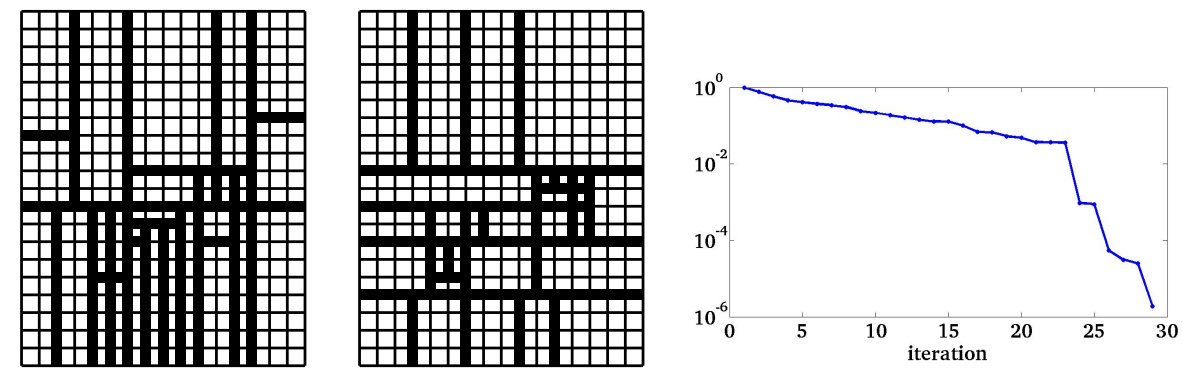

Figure 20: Test 5: strategy 3 when the two exact coefficients have the same zonation. Zonations for $S$ and $T$ after 28 iterations (left and center) and variation of the misfit function during the iterations (right).

We apply now strategy 3 to the case with exact coefficients having different zonations (see Figure 11). As before, the algorithm is initialized with a single zonation for each coefficient (figure 12). Figure 21 shows the variation of the computed coefficients during the iterations. Figure 19 shows the variation of the computed coefficients during the iterations. Figure 22 shows zonations for $S$ and $T$ after 12 iterations and the variation of the normalized misfit function during the iterations. The algorithm identifies $S$ and $T$ after 12 iterations.

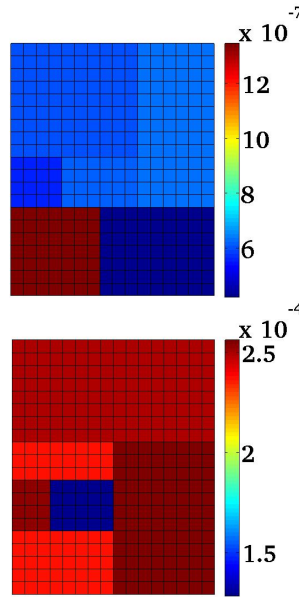

Iteration 6

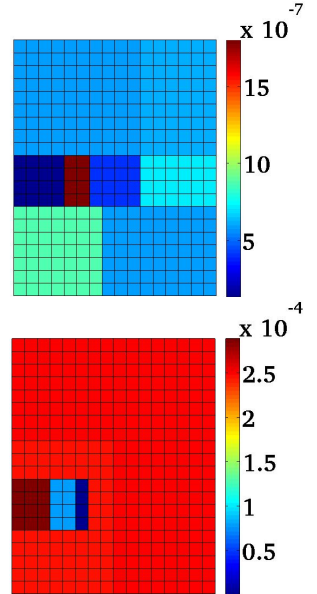

Iteration 8

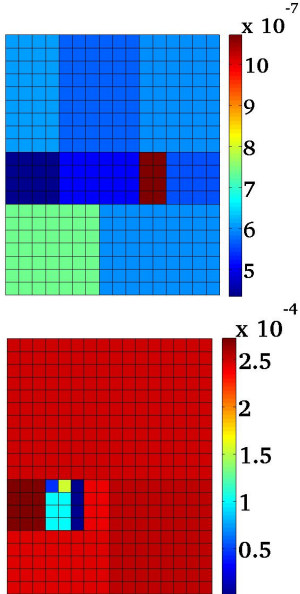

Iteration 11

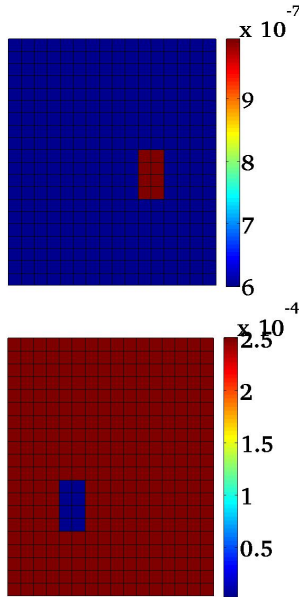

Iteration 12

Figure 21: Test 6: coefficients $S$ (on the left) and $T$ (on the right) computed during the iterations using strategy 3 , when exact $S$ and $T$ have different zonations.

\subsection{Summary of results}

Results for convergence for different strategies and different exact zonations are summarized in Table 1. They show that strategy 1 works well when both exact coefficients have the same zonation while strategy 3 works well when the two coefficients have different zonations. Strategy 2 works well in both cases. In all cases the smaller sensitivity of the system to $S$ than to $T$ is observed by the higher number of iterations for convergence for $S$ than for $T$, and even in some instances the failure to converge for $S$ while the algorithm was converging for $T$. 

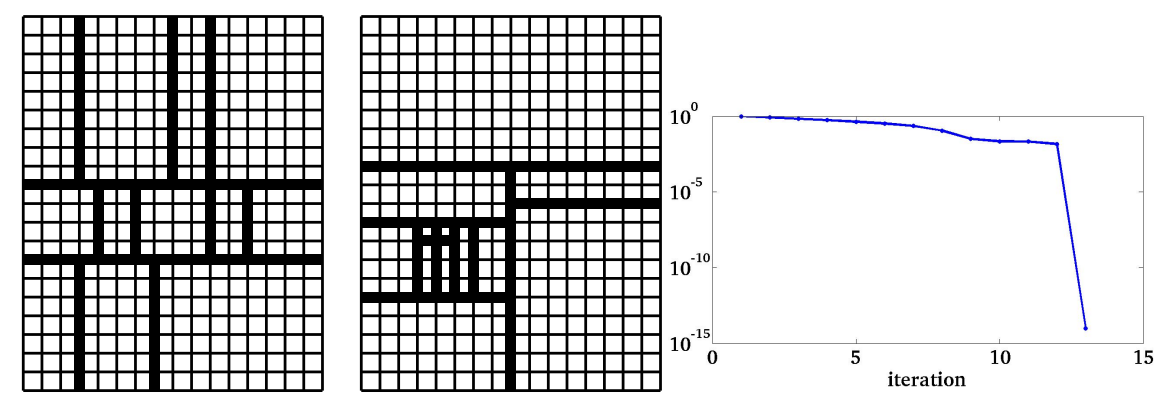

Figure 22: Test 6: strategy 3 when the two exact coefficients have different zonations. Zonations for $S$ and $T$ after 12 iterations (left and center) and variation of the misfit function during the iterations (right).

\begin{tabular}{|c||c|c||c|c||c|c||}
\cline { 2 - 7 } \multicolumn{1}{c|}{} & \multicolumn{2}{c||}{ Strategy 1 } & \multicolumn{2}{c||}{ Strategy 2 } & \multicolumn{2}{c||}{ Strategy 3 } \\
\cline { 2 - 7 } \multicolumn{1}{c|}{} & $S$ & $T$ & $S$ & $T$ & $S$ & $T$ \\
\hline \hline Same exact zonation for $S$ and $T$ & 16 & 14 & 24 & 20 & fails & 25 \\
\hline Different exact zonations for $S$ and $T$ & fails & 17 & 29 & 10 & 12 & 12 \\
\hline
\end{tabular}

Table 1: Number of iterations for convergence of $S$ and $T$ for different strategies and different exact zonations.

\subsection{Influence of the number of measurements}

Because in more realistic cases, we do not have measurements in each cell of the computing mesh, we study the behaviour of the algorithm with respect to a diminution of the number of measurements. We consider the two cases where the parameters have the same or different zonations. For each case we apply the more efficient strategy that are strategy 1 for the first case and strategy 3 for the second case. Two tests are investigated for each case: in a first test we take one observation point out of 2 cells and in a second test we take one observation point out of four cells as shown in Figure 23. We do not decrease the time sampling of data, because the physical properties of our model correspond to a situation of a saturated porous media where the parameters are not time dependent.
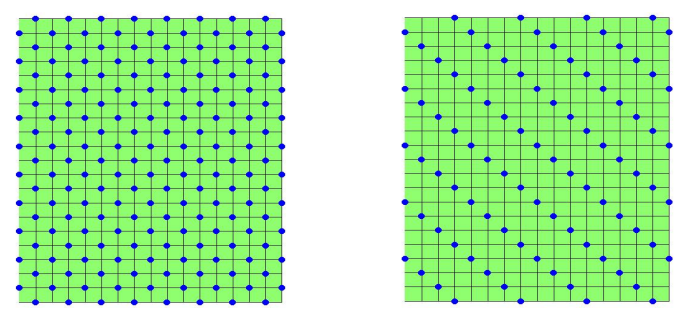

Figure 23: Points of observations: every other point (left), one point out of four (right).

In order to evaluate the fit to the data achieved by the algorithm with different number of 
observation points, we introduce the percentage of explained data

$$
1-\sqrt{\frac{\sum_{i, j}\left|\Phi^{c a l}\left(x_{j}, t_{i}\right)-\Phi_{i j}^{o b s}\right|^{2}}{\sum_{i, j}\left|\Phi_{i j}^{o b s}\right|^{2}}} .
$$

\section{Case of strategy 1 with exact coefficients having the same zonation (Fig. 6)}

In test 7 we use one observation point out of 2 cells as in Figure 23 left while in test 8 we use one observation point out of 4 cells as in Figure 23 right. Figure 24 and Figure 25 show for tests 7 and 8 the values of the obtained coefficients and the decrease of the misfit function when iterating. As before it was easier to recover $T$ than $S$, but the zonations for both coefficients was well identified though the exact values for exact $S$ were not obtained as expected. The results obtained for these two tests are summarized in Table 2.
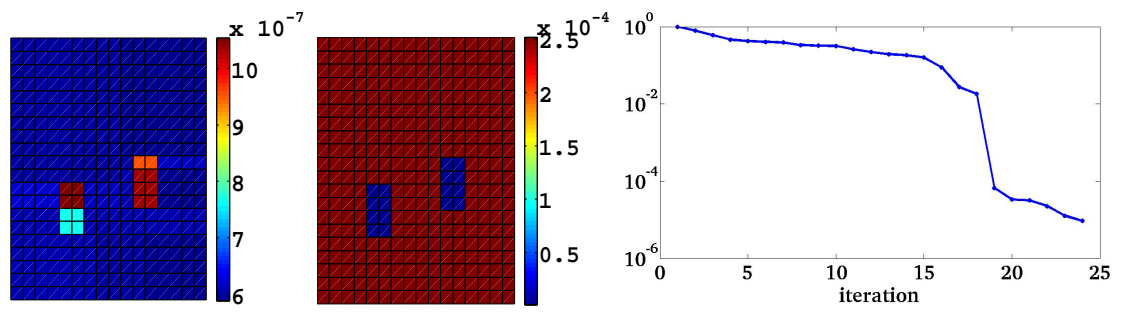

Figure 24: Test 7: strategy 1, when exact $S$ and $T$ have the same zonation. Coefficients $S$ (left) and $T$ (center) computed after 24 iterations and variation of the misfit function (right). Observation points shown in Figure 23 left.

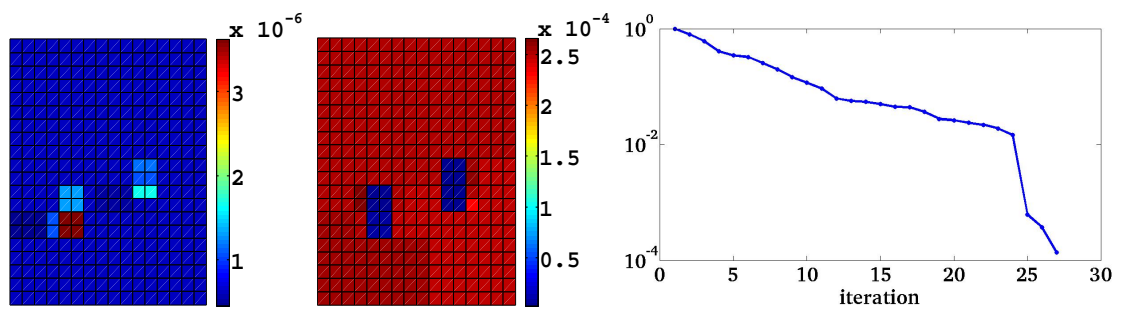

Figure 25: Test 8: strategy 1, when exact $S$ and $T$ have the same zonation. Coefficients $S$ (left) and $T$ (center) computed after 26 iterations and variation of the misfit function (right). Observation points shown in Figure 23 right. 


\begin{tabular}{|c||c||c|}
\hline & $\begin{array}{c}\text { 1 observation point } \\
\text { out of } 2 \text { cells }\end{array}$ & $\begin{array}{c}\text { 1 observation point } \\
\text { out of } 4 \text { cells }\end{array}$ \\
\hline \hline Final number of iterations & 24 & 26 \\
\hline Final number of zones & 24 & 26 \\
\hline$J^{\text {opt }}$ & $6.6710^{-5}$ & $1.45 \quad 10^{-2}$ \\
\hline Percentage of explained data & 0.99 & 0.97 \\
\hline
\end{tabular}

Table 2: Results with a reduced number of measurements, strategy 1 and case of exact coefficients with the same zonation

Case of strategy 3 with exact coefficients having different zonations (Fig. 11) In strategy 3 we use different zonations for each coefficient. In test 9 we use one observation point out of 2 cells as in Figure 23 left while in test 10 we use one observation point out of 4 cells as in Figure 23 right. Figure 26 and Figure 27 show for tests 9 and 10 the values of the obtained coefficients and the decrease of the misfit function when iterating. Results are summarized in Table 3. As before it was easier to recover $T$ than $S$, but in test 9 the inclusions for each coefficient were well identified with values for close to the exact ones. But for test 10 the situation deteriorates significantly, especially for $S$.

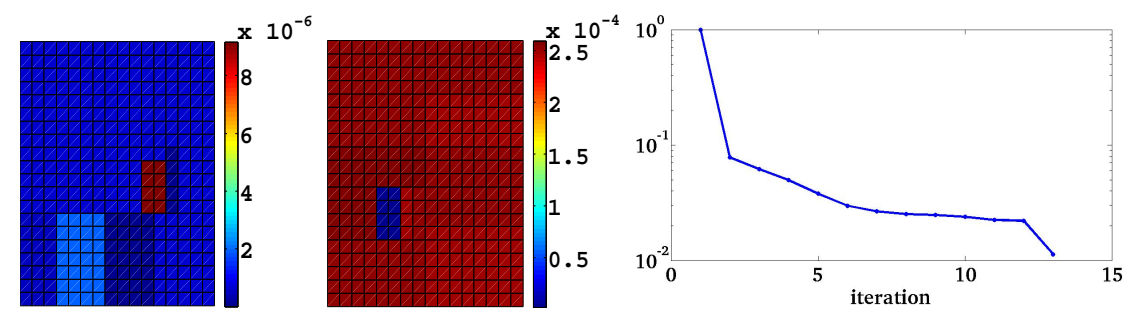

Figure 26: Test 9: strategy 3, when exact $S$ and $T$ have different zonations. Coefficients $S$ (left) and $T$ (center) computed after 14 iterations and variation of the misfit function (right). Observation points shown in Figure 23 left.
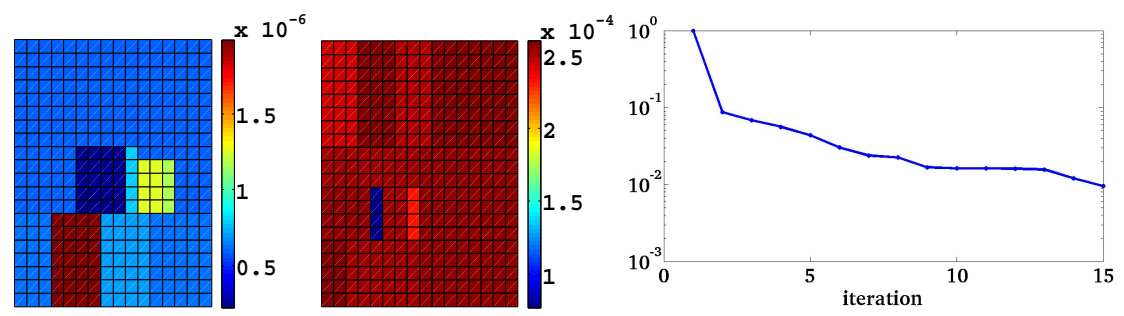

Figure 27: Test 10: strategy 3, when exact $S$ and $T$ have different zonations. Coefficients $S$ (left) and $T$ (center) computed after 15 iterations and variation of the misfit function (right). Observation points shown in Figure 23 right. 


\begin{tabular}{|c||c||c|}
\hline & $\begin{array}{c}1 \text { observation point } \\
\text { out of } 2 \text { cells }\end{array}$ & $\begin{array}{c}1 \text { observation point } \\
\text { out of } 4 \text { cells }\end{array}$ \\
\hline \hline Final number of iterations & 14 & 15 \\
\hline Final number of zones & 14 & 15 \\
\hline$J^{\text {opt }}$ & $1.1310^{-2}$ & $1.2010^{-2}$ \\
\hline Percentage of explained data & 0.98 & 0.93 \\
\hline
\end{tabular}

Table 3: Results with a reduced number of measurements, strategy 1 and case of exact coefficients with the same zonation

\section{Conclusion}

We presented a method for the simultaneous estimation of the storage coefficient and the hydraulic transmissivity in a confined aquifer. These two coefficients are assumed to be functions piecewise constant in space and constant in time. The unknowns of the inverse problem are the zonations for each coefficient and their values in each zone. The two coefficients may or may not have the same zonation. The method is a generalization of the adaptive parameterization algorithm guided by refinements indicators in [1] introduced for scalar parameters to the case of a vector parameter.

We consider three variants corresponding to three strategies for choosing the refinement indicators. The first two strategies construct the same zonation for the two coefficients while the last one construct different zonations for the coefficients. Strategy 1 is efficient for the case when the two exact coefficients have the same zonation while strategy 3 is efficient when the two exact coefficients have different zonations. Strategy 3 was working well in both cases while a little slower than the two other strategies when they work well. Obviously the results depend on the sensitivity of the system to each coefficient.

These first results should be completed with more experiments exploring more configurations of the inclusions we are looking for. They could be improved by combining the method that we presented with coarsening indicators in order to decrease the number of zones produced, thus making minimization steps easier. One should also note that our method can be applied for a problem with more than two coefficients to identify.

\section{References}

[1] Hend Ben Ameur, Guy Chavent, and Jérôme Jaffré. Refinement and coarsening indicators for adaptive parametrization: application to the estimation of hydraulic transmissivities. Inverse Problems, 18(3):775, 2002.

[2] Hend Ben Ameur, François Clément, Pierre Weis, and Guy Chavent. The multidimensional refinement indicators algorithm for optimal parameterization. Journal of Inverse and IllPosed Problems, 16(2):107-126, 2008.

[3] G. Chavent and J. Liu. Multiscale parametrization for the estimation of a diffusion coefficient in elliptic and parabolic problems. In A. El Jai and M. Amouroux, editors, 5th IFAC Symposium on Control of Distributed Parameter Systems, Perpignan, June 26-29, 1989, pages 315-324. Université de Perpignan, France, 1989.

[4] Guy Chavent. On the theory and practice of non-linear least-squares. Advances in Water Resources, 14(2):55-63, 1991. 
[5] Guy Chavent. Nonlinear Least Squres for Inverse Problems: Theoretical Foundations and Step-by=Step Guide for Applications. Springer, 2009.

[6] Guy Chavent and Liu Jun. Multiscale parameterization for the estimation of a diffusion coefficient in elliptic and parabolic problems. In M. Amouroux and A. El Jai, editors, 5th IFAC Symposium on Control of Distributed Parameter Systems, 26-29 June 1989, Perpignan, France, pages 193-202. IFAC, The International Federation of Automatic Control, 1990.

[7] R. L. Cooley. Incorporation. Water Resources Research, 13:318-324, 1982.

[8] G De Marsily, A Buoro, et al. 40 years of inverse problems in hydrogeology. Comptes Rendus de l'Academie des Sciences Series IIA Earth and Planetary Science, 329(2):73-87, 1999.

[9] A.A. Grimstad, T. Mannseth, G. Næ vdal, and H. Urkedal. Adaptive multiscale permeability estimation. Computational Geosciences, 7:1-25, 2003.

[10] Mohamed Hayek and Philippe Ackerer. An adaptive subdivision algorithm for the identification of the diffusion coefficient in two-dimensional elliptic problems. Journal of Mathematical Modelling and Algorithms, 6(4):529-545, 2007.

[11] Mohamed Hayek, Philippe Ackerer, and Éric Sonnendrücker. A new refinement indicator for adaptive parameterization: Application to the estimation of the diffusion coefficient in an elliptic problem. Journal of Computational and Applied Mathematics, 224(1):307-319, 2009.

[12] C Lemaréchal, E Panier, and J. Ch. Gilbert. Modulopt, N2QN1. https://who.rocq.inria.fr/Jean-Charles.Gilbert/modulopt/

[13] J. Liu. A multiresolution method for distributed parameter estimation. SIAM J. Sci. Comput., 14:389-405, 1993.

[14] Ne-Zheng Sun. Inverse problems in groundwater modeling. Kluwer Academic Publishers, 1994.

[15] Clifford I Voss. A finite-element simulation model for saturated-unsaturated, fluid-densitydependent ground-water flow with energy transport or chemically-reactive single-species solute transport, volume 84. US Geological Survey, 1984.

[16] William W-G Yeh. Review of parameter identification procedures in groundwater hydrology: The inverse problem. Water Resources Research, 22(2):95-108, 1986.

[17] William W-G Yeh and Young S Yoon. Aquifer parameter identification with optimum dimension in parameterization. Water Resources Research, 17(3):664-672, 1981. 


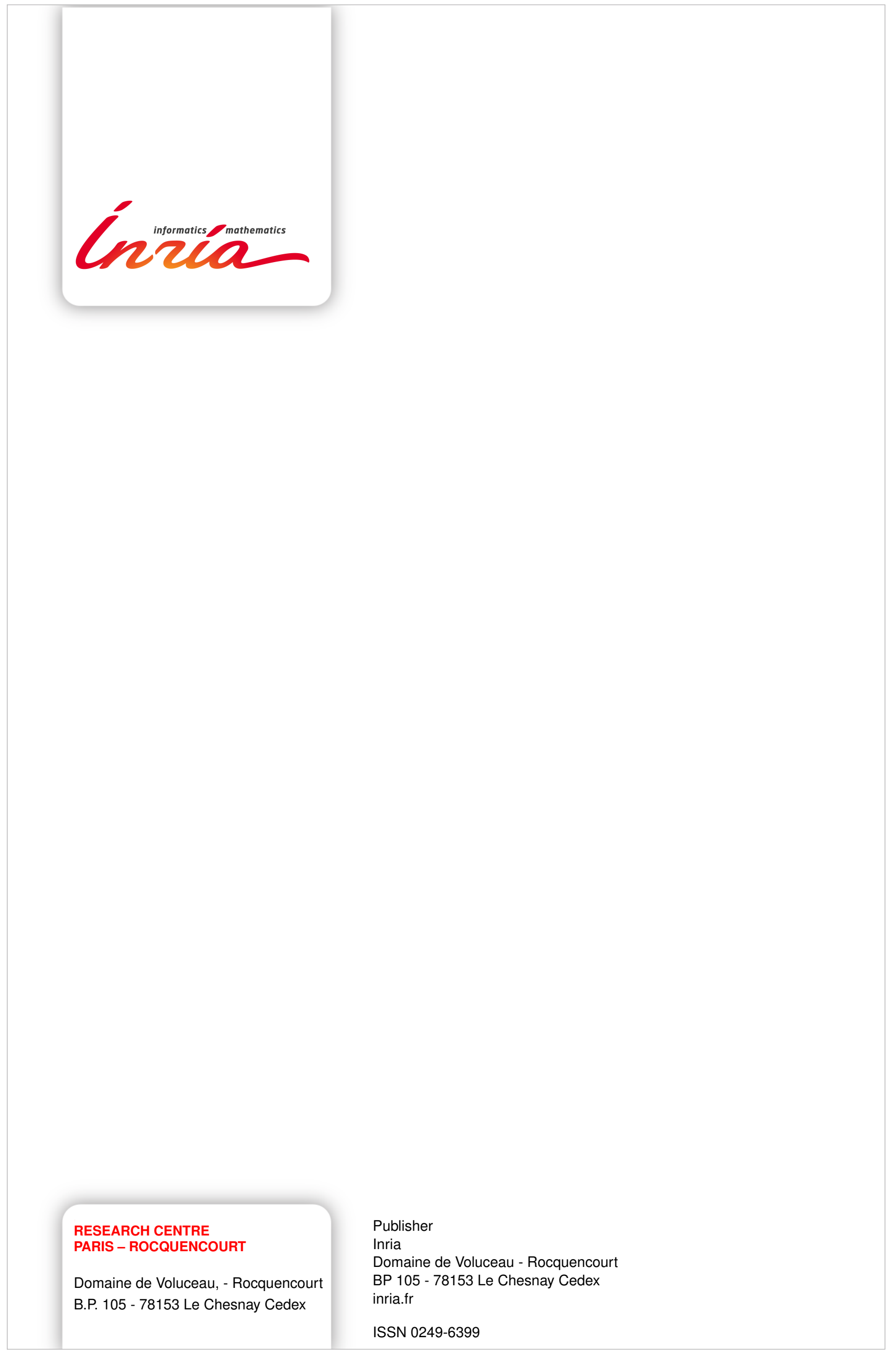

